\title{
Reforming teaching methods by integrating dental theory with clinical practice for dental students
}

\author{
Wei Wang ${ }^{\text {Corresp., Equal first author, } 1}$, Xuewei Bi ${ }^{\text {Equal first author, } 2,3}$, Yuhe Zhu ${ }^{1}$, Xiaoming Li ${ }^{\text {Corresp. } 2,3}$ \\ 1 Department of General Dentistry, School of Stomatology, China Medical University, Shenyang, China \\ Beijing, China \\ 3 Beijing Advanced Innovation Center for Biomedical Engineering, Beihang University, Beijing, China \\ Corresponding Authors: Wei Wang, Xiaoming Li \\ Email address: wwang75@cmu.edu.cn, lixm@buaa.edu.cn
}

Background. Transitioning from theoretical medicine to clinical practice is both an important and difficult process in the education of dental students. Thus, there is an urgent need for teaching methods that can improve the ability of dental students to integrate dental theory with clinical practice.

Methods. First, we conducted problem-based learning training, based on real clinical cases, for dental students. The students were then assigned to dentist/patient roles to rehearse and perform simulated clinical scenarios. Finally, questionnaires, clinical patient care scores, and performance assessments were utilized to evaluate and compare the effectiveness of this training with that of traditional teaching methods.

Results. The abilities of the students after using this reformed teaching method markedly increased in terms of the treatment of and communication with patients. Among the 30 enrolled students, 29 liked the method, found it time-efficient, and believed that it could help enhance their problem-solving confidence and interest in prosthodontics. They also believed that this teaching method could help them gain a good understanding of related theoretical material, generally thought that the reformed teaching method was more valuable than the traditional approach, and would like to introduce it to others.

Conclusion. After the teaching method change, the students not only achieved better scholastically, but also demonstrated greater accuracy in diagnosing the conditions of patients and formulating treatment plans. It was much easier for them to obtain recognition from patients, indicating that this method is effective for dental students. 


\section{Reforming teaching methods by integrating dental theory with clinical 2 practice for dental students}

3 Wei Wang ${ }^{1, *}$, Xuewei $\mathrm{Bi}^{2,3}$, Yuhe Zhu ${ }^{1,}$, Xiaoming $\mathrm{Li}^{2,3, *}$

41 Department of General Dentistry, School of Stomatology, China Medical University, Shenyang, China

52 Key Laboratory for Biomechanics and Mechanobiology of Ministry of Education, School of Biological Science

6 and Medical Engineering, Beihang University, Beijing, China

73 Beijing Advanced Innovation Center for Biomedical Engineering, Beihang University, Beijing, China

9 Wei Wang and Xuewei Bi are co-first authors of this article.

10 Wei Wang and Xiaoming Li are Corresponding authors.

* Corresponding author. E-mail address: wwang75@cmu.edu.cn (W. Wang), lixm@buaa.edu.cn (X. Li)

\section{Abstract}

Background. Transitioning from theoretical medicine to clinical practice is both an important and difficult process in the education of dental students. Thus, there is an urgent need for teaching methods that can improve the ability of dental students to integrate dental theory with clinical practice.

Methods. First, we conducted problem-based learning training, based on real clinical cases, for dental students. The students were then assigned to dentist/patient roles to rehearse and perform simulated clinical scenarios. Finally, questionnaires, clinical patient care scores, and performance assessments were utilized to evaluate and compare the effectiveness of this training with that of traditional teaching methods.

Results. The abilities of the students after using this reformed teaching method markedly increased in terms of the treatment of and communication with patients. Among the 30 enrolled students, 29 liked the method, found it timeefficient, and believed that it could help enhance their problem-solving confidence and interest in prosthodontics. They also believed that this teaching method could help them gain a good understanding of related theoretical material, generally thought that the reformed teaching method was more valuable than the traditional approach, and would like to introduce it to others. Conclusion. After the teaching method change, the students not only achieved better scholastically, but also demonstrated greater accuracy in diagnosing the conditions of patients and formulating treatment plans. It was much easier for them to obtain recognition from patients, indicating that this method is effective for dental students.

\section{Introduction}

Transitioning from a medical theoretical education to clinical practice is an important, but difficult, process for dental students. Clinical practice is usually conducted in the last year of study, and some do not ever participate in clinical practice (D Xu et al, 2010). During traditional clinical practice, students cannot actually perform operations on patients, and do not have enough time to communicate with patients in the clinic. They can only observe how teachers 
37 highlights the challenge of merging theoretical knowledge and clinical practice in dental education. Dental students do not know how to respond when faced directly with patients. They cannot apply their theoretical knowledge to clinical diagnosis and treatment, which could easily lead to medical disputes, especially when the doctor-patient relationship is already strained. Therefore, there is an urgent need for an effective teaching method that can integrate dental theory with clinical practice when educating dental undergraduate students.

Problem-based learning (PBL) training is designed to use a high-authenticity task, emphasizing the study of learning in complex and meaningful problem scenarios. Learners can solve problems through self-exploration and cooperation, and can learn scientific knowledge based on the problem. Students develop both the skills to solve the problem and self-learning abilities. PBL training has been applied in medical education for more than 40 years (Edward \& Thompson, 2013). Previous studies have shown that medical students who underwent PBL training had better results in medical licensing examinations and clinical practice, and showed a better understanding of clinical problems and a capacity for self-learning than students who underwent traditional teaching (Blake, Hosokawa \& Riley, 2000; Hoffman et al., 2006; Okubo et al., 2012; Khan et al., 2007). Although PBL training has been widely used, Kinkade et al.'s research showed that its application in American medical colleges has declined (Kinkade et al., 2005), mainly because teachers realized that PBL training preparation was time consuming and required the use of more staff. For that reason, the practicality of spending more human resources to conduct PBL training has been questioned (Distlehorst et al., 2005; Colliver, 2000; Farnsworth, 1994; Kirschner, Sweller \& Clark, 2006). China, with its rising educational reform, has been gradually introducing PBL training in medical education. Some researchers have investigated the use of PBL training in Chinese medical colleges, and found that PBL training had been applied in 43 medical colleges, and its utilization rate in the pre-clinical curriculum was about 50\% (Fan, Kosik \& Tsai, 2014). However, there have been very few reports showing the effectiveness of PBL training in dental undergraduate education. Above all, a reform of dental education that can better combine theoretical knowledge with clinical practice is needed (Du et al,2010). Traditional dental education has always used lecturing as the main teaching method, with an emphasis on acquiring basic theoretical knowledge. Although this teaching method can help students grasp knowledge points and holistic theory, it cannot track their initiative. Therefore, students lack the ability to practically apply their knowledge, the capacity for self-learning, and clinical reasoning experience (Wang, J. et al, 2010). It is very difficult for students to link clinical practice with theoretical knowledge, or to apply material from a lecture to solve clinical problems when faced with real patients. Although many educational models have been proposed for dental students, the critical transition from theoretical teaching to clinical practice training remains unacknowledged (Prince, 2000). Since specialized teachers in medical colleges often concurrently work as consultants in clinics, their dedicated teaching time is very limited. It is impractical to abolish the existing teaching method completely and replace it entirely with the PBL teaching method. It is more appropriate to find a compromise between these two teaching methods, one that is both practical and better at fulfilling the professional training requirements for dental students (S Baozhi et al, 2003). Furthermore, from the feedback of students, we found that those who had been taught using traditional methods

71 lacked not only sufficient capability in clinical practice, but also a satisfactory ability in communicating with patients.

72 These students overlooked some necessary details, such as their appearance, attitude, tone and rate of speech, way of 73 expression, etc., when communicating with patients (Du et al, 2013), and they did not show enough consideration to 
74 their patients before and during treatments. All these factors may cause medical disputes and patient mistrust of 75 dentists, and they may also have negative effects on the formulation of an accurate diagnosis and treatment plan.

76 Given the factors above, we designed a clinical simulation PBL training method to both improve the ability of dental 77 undergraduate students to integrate dental theory with clinical practice, as well as enhance their professional skills, 78 and we compared this method's efficacy with that of the traditional teaching method.

79 Materials \& Methods

80 Ethics Statement

81 A standard written informed consent procedure was included in the protocol, and was reviewed and approved by the 82 Ethics Committee of China Medical University. All the participants were over the age of 18 years, and gave their 83 written consent after the nature of the study had been fully explained. The research was approved by the Ethics

84 Committee of China Medical University, and conducted in full accordance with the World Medical Association 85 Declaration of Helsinki.

\section{Teaching objects and grouping}

87 PBL training was first conducted based on real clinical cases. To investigate whether the clinical-simulation PBL training method was applicable to dental undergraduate teaching, we divided 60 students into two groups, 30 of whom underwent PBL training while the other 30, as the control group, received traditional teaching. Questionnaires, clinical patient care scores, and performance assessments were utilized to evaluate the effectiveness of PBL training when compared with that of traditional teaching. Thirty fifth-grade undergraduates from the School of Stomatology, China Medical University, participated in PBL training in 2017: 11 males and 19 females. Another 30 undergraduate students from the same grade who underwent normal class teaching without PBL training were set as the control group: 11 males and 19 females. The 30 students in each group were further divided into five subgroups, with six in each subgroup. Each subgroup was comprised of members with different cognitive characteristics, aptitudes, and personalities. There were distinct differences among group members, but the overall study ability level of each subgroup was consistent.

\section{Selection of four clinical cases for PBL training}

The four selected clinical cases covered the basic elements of prosthodontics, including dental defect repair, fixed partial denture repair, removable partial denture repair, and complete denture repair. Prostheses were applied as the main treatments for all cases. However, before the final installation of the prostheses, pre-treatments such as dental treatment of oral medicine, periodontal treatment, oral extraction surgery, etc., had been performed.

\section{Design of the training protocol}

The students were given enough time for systematic discussion and analysis of differential diagnoses, pre-treatment plans, and restoration treatment plans of the four cases in their allocated groups. They were then asked to devise a reasonable and comprehensive treatment plan. The students undergoing PBL training conducted a simulated clinical diagnosis and treatment, taking turns to play the roles of doctor and patient, while the remaining students in the same group pointed out errors and proposed suggestions. All students repeated the practice until they received satisfactory evaluations from teachers and student judges.

110 Teachers provided the PBL problems related to the four clinical cases one week in advance, and then announced the four cases to the students. Each group of students worked as a team to search the relevant literature, and then submitted 
112 a summary report. Each team member was allocated an approximately equal amount of work according to their own

113 characteristics after an internal group discussion. When any team had questions, the teacher would provide necessary 114 guidance.

115 After each student had worked independently, all team members were asked to exchange information to discuss the

116 problem-solving process, and then to draw conclusions. Teachers encouraged discussions, ensuring that each group

117 stuck closely to the PBL theme, and re-examined any previous errors. The group members continued to revise their

118 written reports with any new relevant literature on the problems posed by the teachers.

119 The teachers instructed the students to summarize their experiences and deficiencies throughout the training process.

120 They also evaluated the students' independent learning and collaborative abilities.

121 After listening to the presentations of all the groups, the teachers gave comments on the answers to the PBL questions, 122 and then provided any necessary corrective suggestions. Professional treatment advice was also given to each group 123 based on their treatment plan.

\section{Evaluations}

125 Questionnaires, clinical patient care scores, and performance assessments were utilized to evaluate the effectiveness of the PBL training when compared with that of the traditional teaching approach. After the training, a survey of the students was taken, including their responses to changes in their general abilities or skills, changes in their treatment or communication abilities, the cognition of the teaching method, etc.

Second, teachers selected five real patients in the clinic, and two groups (the training group and control group) of students independently admitted them. The patients selected needed dental defect repair, fixed partial denture repair, removable partial denture repair, and complete denture repair. The teachers filled out the clinical case score sheet (total 100 points, table 1), which included evaluations on the students' abilities in communicating with patients, their auxiliary examinations before operation and differential diagnosis, the design and description of their treatment plans, etc. The teachers scored each student's performances for all items on the sheet, and we compared the scores of the PBL training group with those of the control group.

Finally, a paper examination on prosthodontics was used to investigate the learning outcomes of the two groups of students. The types of examination questions were multiple choice, fill in the blank, short answers, and case analysis questions. Full marks for the examination was 100 points, with 60 points or less considered a failure, between 60 and 90 points considered a pass, and 90 or more points considered excellent.

140 The test scores of the groups were expressed as mean value \pm standard deviation. Statistical calculations were done

141 with SPSS (Chicago, IL, USA) 21.0 Windows software. T-test was used to analyze differences of the data between

142 the groups. A $p<0.05$ was regarded as significant difference.

\section{Results}

144 After the end of the clinical simulation PBL training curriculum, students were surveyed to evaluate the effect of the 145 training. Figures 1 to 5 show various aspects of the questionnaires, such as student responses to questions about 146 changes in their general abilities or skills, changes in their special abilities of treating diseases or communicating with 147 patients, the cognition of the teaching method, etc. The results showed that the general abilities or skills of the students 148 after the PBL training had markedly increased (Figure 1), including their abilities to independently search literature, 149 their comprehensive and logical analysis skills, their teamwork ability, and their curiosity and exploratory desire of 
150

151

152

153

154

155

156

157

158

159

160

161

162

163

164

165

166

167

168

169

170

171

172

173

174

175

176

177

178

179

180

181

182

183

184

185

186 professional knowledge. Moreover, their special abilities in treating diseases or communicating with patients, including understanding indications for repair, correctly diagnosing diseases, developing treatment plans, quickly and accurately recognizing the patient's condition, communicating with and understanding patients, had notably increased (Figure 2). This teaching method was highly regarded by the students (Figure 3). Among the 30 students, 29 liked this teaching method. Twenty-eight students considered this method an efficient use of time. Twenty-six believed that this teaching method could help enhance their problem-solving confidence. Twenty-seven students believed that this teaching method could increase their interest in prosthodontics, while 25 believed that this teaching method could help them gain a better theoretical knowledge of prosthodontics. Twenty-eight students were keen to introduce this teaching method to others. Twenty-nine students believed that the value of this teaching method was greater than that of the traditional teaching approach (Figure 4).

The score sheets for clinical practice (Table 1) were designed to evaluate the students' clinical performance, including meeting the requirements for appearance; their attitude to the patients, their ability to communicate with patients, diagnose diseases, make a differential diagnosis, perform auxiliary examinations and operational examinations; devise early restoration treatment plans before making the prosthesis; patients' satisfaction; and so on. Table 2 shows that students who underwent clinical-simulation PBL training received a score of $88.90 \pm 2.29$, which was significantly higher than the score of $67.13 \pm 2.20$ received by the students who had not undergone the training $(\mathrm{p}<0.05)$. This suggests that the clinical-simulation PBL training method was very helpful for students in the clinical management of patients. More specifically, the sub-scores of items 3 and 10 were respectively $14.17 \pm 0.38$ and $9.53 \pm 0.63$, showing significant improvement as compared with those $(9.27 \pm 1.46$ and $6.80 \pm 0.41)$ of the students in the control group $(\mathrm{p}<0.05)$.

Figure 5 shows the prosthodontics examination scores of the clinical-simulation PBL training group and the control group. The average score of the training group was 82.80 points, and the pass rate and the excellent rate were respectively $90 \%$ and $40 \%$. The average score of the control group was 74.33 points, and the pass rate and the excellent rate were respectively $70 \%$ and $23.33 \%$.

\section{Discussion}

Learning clinical reasoning is complex, as it includes the application of professional knowledge and the accumulation of experience from actual clinical cases. Traditional teaching methods only focus on theoretical knowledge and lack clinical reasoning training, without a link between theoretical knowledge and clinical cases. After students graduate, their theoretical knowledge cannot be adequately applied in clinical practice, and they do not have enough selfconfidence nor communication abilities when faced with patients.

With rapid developments in dental medical technology, dentists must have the ability to independently learn new information and skills. Dentists should also have a good ability to communicate, in order to avoid misunderstandings with their patients. A single traditional teaching approach for dental education is no longer applicable for dental students. It is necessary to improve the existing teaching methods and add innovative methods.

In this study, we proposed simulated clinical PBL training on the basis of traditional teaching methods. First, several typical clinical cases were chosen and compiled into templates. Students collected the necessary information through literature review and then discussed within small groups to solve clinical problems, formulate rational treatment plans,

Peer) reviewing PDF | (2019:06:38500:3:0:NEW 15 Dec 2019) 
187

188

189

190

191

192

193

194

195

196

197

198

199

200

201

202

203

204

205

206

207

208

209

210

211

212

213

214

215

216

217

218

219

220

221

222

and determine the most suitable treatment for patients. During this PBL training, students were instructed how to apply their basic dental theoretical knowledge to clinical cases. The training provided students with an opportunity to maximize simulated clinical practice, stimulating their self-learning capacities and problem-solving skills when faced with real patients in the clinic.

In addition to being able to apply basic dental theoretical knowledge to clinical cases, a dentist should also have a satisfactory ability in communicating with patients, enabling them to obtain more relevant information about medical history and current diseases, and to formulate accurate diagnosis and treatment plans. Therefore, in the second part of the training, some of the students in the PBL group took turns playing the roles of doctor and patient, conducting simulated clinical diagnosis and treatment, while the other students in the same group acted as judges to point out errors. All students repeated the practice until they received satisfactory evaluations from the teachers and their peers. The results showed that students generally believed that PBL teaching could promote critical thinking ability more than traditional teaching methods, and that this teaching method was very helpful for improving their capacity for selflearning. After the training, students showed improved ability to communicate with patients, greater accuracy in diagnosing patients' conditions and formulating treatment plans, and it was much easier for them to obtain the appreciation of patients. Furthermore, they believed that PBL training could facilitate the comprehensive utilization of various theoretical facts into oral professional and clinical practice, and that the clinical scenario simulation during the PBL training was especially helpful for the improvement of their linguistic skills, logical thinking, and clinical practice ability. Additionally, the results of the prosthodontics examination scores suggested that PBL training deepened students' understanding of the related theoretical knowledge, leading to improved performances. This teaching method was highly regarded by the students, and the results indicate that clinical-simulation PBL is likely to be an effective teaching method for dental undergraduate students.

Through this study, we found many factors that might affect the effectiveness of clinical-simulation PBL training for dental undergraduate students: (i) appropriate clinical case selection, (ii) reasonable proposed problems, (ii) abundant rehearsal and role-play of dentists and patients, and (iv) sufficient preparation, discussion, and practice time. When choosing cases, teachers should fully consider common occurrences in local clinics. For example, patients in prosthodontics clinics often need the consultation of doctors from other oral departments, such as oral medicine, oral periodontal, oral surgery, etc. Therefore, the overall oral health should be considered overall. Second, implementing the core part of PBL training can be difficult. The proposed PBL problems directly influence the effectiveness of student learning, and should be designed to attract the student's interests in understanding the cases. When discussions are restricted to certain issues, teachers should remind students to extend their range of thought, and should ultimately help students find satisfactory answers and develop rational prosthetic treatment plans (Barrows \& Tamblyn, 2003; Hung, 2011; Li et al., 2015). Third, abundant rehearsal and role-play of dentist and patient significantly enhance the effectiveness of the clinical-simulation PBL training for dental undergraduate students. Cultivation of the students' communication and understanding has always been emphasized in modern higher education. For dental students, abundant rehearsal and role-play may help them comprehensively understand patients, which is crucial for achieving satisfactory diagnosis and making treatment plans. Fourth, during training, sufficient time should be given to teachers 
223 to ensure they have enough time to instruct students properly, and to students so that they have ample time to access

224 relevant information, have full discussions, and enough repeated practice to obtain good results.

225 Although satisfactory results have been obtained in this study, we noticed that there were still some challenges in 226 conducting clinical-simulation PBL training in dental schools. There was only limited funding for dental education 227 and educational research. Most of the teachers who are normally dentists did not have enough specific time allocated 228 for clinical-simulation PBL training. Many students lacked adaptability to this training method. However, we believe 229 that with its increasing recognition and optimization by dentists and students, clinical-simulation PBL training may 230 become more widely applied in dental education.

231 Moreover, some limitations of this study should be mentioned. Firstly, it would be better if the number of study 232 subjects were larger. Secondly, a systematic and standardized evaluation system should be established in the further study to reflect the effect of reformed teaching.

\section{Conclusion}

237 In this study, clinical-simulation PBL training was designed to integrate dental theory with clinical practice for dental students. PBL training was first conducted based on real clinical cases. Students had the opportunity to repeatedly participate in role-play as dentists and patients to simulate clinical scenarios. The results showed that students generally believed that PBL teaching could promote their critical thinking ability more than traditional teaching methods, and that this teaching method was very helpful in improving the capacity for self-learning. After the training, the students showed improved ability to communicate with patients, greater accuracy in diagnosing patient conditions and formulating treatment plans, and it was much easier for them to obtain the acknowledgement of the patients. Furthermore, PBL training was considered to facilitate the comprehensive utilization of various theoretical facts into oral professional and clinical practice, and clinical scenario simulation during the PBL training was especially helpful for the improvement of linguistic skills, logical thinking, and clinical practice ability. The results of the prosthodontics examination scores suggested that PBL training can also deepen students' understanding of related theoretical knowledge, leading to improved performances. Overall, this teaching method was highly regarded by the students. These results indicated that clinical-simulation PBL is likely to be an effective teaching method for dental undergraduate students.

\section{References}

252 Blake RL, Hosokawa MC, Riley SL. 2000. Student performances on Step 1 and Step 2 of the United States Medical

253 Licensing Examination following implementation of a problem-based learning curriculum. Academic Medicine 254 75(1):66-70

255 Brieger WR. 1981. Problem-based learning: An approach to medical education. International Journal of Health 256 Education 24(3):204-205

257 Colliver JA. 2000. Effectiveness of problem-based learning curriculum: research and theory. Academic Medicine 258 75(3):259-266

259 Distlehorst LH, Dawson E, Robbs RS, Barrows HS. 2005. Problem-based learning outcomes: The glass half-full. 
261 Du GF, Li CZ, Shang SH, Xu XY, Chen HZ, Zhou G. 2013. Practising case-based learning in oral medicine for dental 262 students in China. European Journal of Dental Education 17(4):225-228

263 Du XY, Emmersen J, Toft E, Sun B. 2013. PBL and critical thinking disposition in Chinese medical students: - A 264 randomized cross-sectional study. Journal of Problem Based Learning in Higher Education 1(1):72-83

265 Edward CK, Thompson TL. 2013. Can PBL group assignment affect examination performance? Medical Science 266 Educator 23(2):244-249

267 Fan APC, Kosik RO, Tsai TCC, Cai QL, Xu GT, Guo L, Su TP, Wang SJ, Chiu AWH, Chen Q. 2014. A snapshot of 268 the status of problem-based learning (PBL) in Chinese medical schools. Medical Teacher 36(7):615-620

269 Hoffman K, Hosokawa M, Blake R, Headrick L, Johnson G. 2006. Problem based learning outcomes: Ten years of 270 experience at the University of Missouri-Columbia School of Medicine. Academic Medicine 81(7):617-625

271 Hung W. 2011. Theory to reality: a few issues in implementing problem-based learning. Education Technology

272 Research and Development 59(4):529-552

273 Khan H, Taqui AM, Khawaja MR, Fatmi Z. 2007. Problem-based versus conventional curricula: Influence on

274 knowledge and attitudes of medical students towards health research. PLOS ONE 2(7):e632-637

275 Kinkade SA. 2005. A snapshot of the status of problem-based learning in U.S. medical schools, 2003-04. Academic

276 Medicine 80(3):300-301

277 Kirschner PA, Sweller J, Clark RE. 2006. Why minimal guidance during instruction does not work: An analysis of 278 the failure of constructivist, discovery, problem-based, experiential, and inquiry-based teaching. Educational 279 Psychologist 41(2):75-86

280 Li XM, Zhao F, Pu F, Liu HF, Niu XF, Zhou G, Li DY, Fan YB, Feng QL, Cui FZ, Watari F. 2015. A multidisciplined 281 teaching reform of biomaterials course for undergraduate students. Journal of Science Education and Technology 282 24(6):735-746

283 Montani JP, Antic V, Summers RL, Coleman TG, Headrick L, Johnson G. 1998. Problem based learning using 284 computer simulations. Federation of American Societies for Experimental Biology Journal 12(4):A17

285 Nair H, Nokes DJ, Gessner BD, Dherani M, Madhi SA, Singleton RJ, O'Brien KL, Roca A, Wright PF, Bruce N, 286 Chandran A, Theodoratou E, Sutanto A, Sedyaningsih ER, Ngama M, Munywoki PK, Kartasasmita C, Simões EA, 287 Rudan I, Weber MW, Campbell H. 2010. Global burden of acute lower respiratory infections due to respiratory 288 syncytial virus in young children: a systematic review and meta-analysis. Lancet 375(9275):1545-1555

289 Okubo Y, Ishiguro N, Suganuma T, Nishikawa T, Takubo T, Kojimahara N, Yago R, Nunoda S, Sugihara S, Yoshioka 290 T. 2012. Team-based learning, a learning strategy for clinical reasoning, in students with problem-based learning 291 tutorial experiences. Tohoku Journal of Experiment Medicine 227(1):23-29

292 Prince KJAH, van de Wiel MJ, Scherpbier AJJA, van der Vleuten CPM, Boshuizen HPA. 2000. A qualitative analysis 293 of the transition from theory to practice in undergraduate training in a PBL-medical school. Advancein Health Science 294 Education Theory and Practice 5(2):105-116

295 Sun BZ, Zhao YH. 2003. Medical curricula in China and the USA: a comparative study. Medical Teacher 25(4):422296 427 
297 Wang J, Zhang W, Qin L, Zhao J, Zhang S, Gu J, Zhou C. 2010. Problem-based learning in regional anatomy education 298 at Peking University. Anatomical Sciences Education 3(3):121-126 


\section{Figure 1}

Student responses to the questions about the change in their general abilities or skills after the clinical-simulation PBL training

The figure shows that student responses to the questions about the change in their general abilities or skills after the clinical-simulation PBL training 


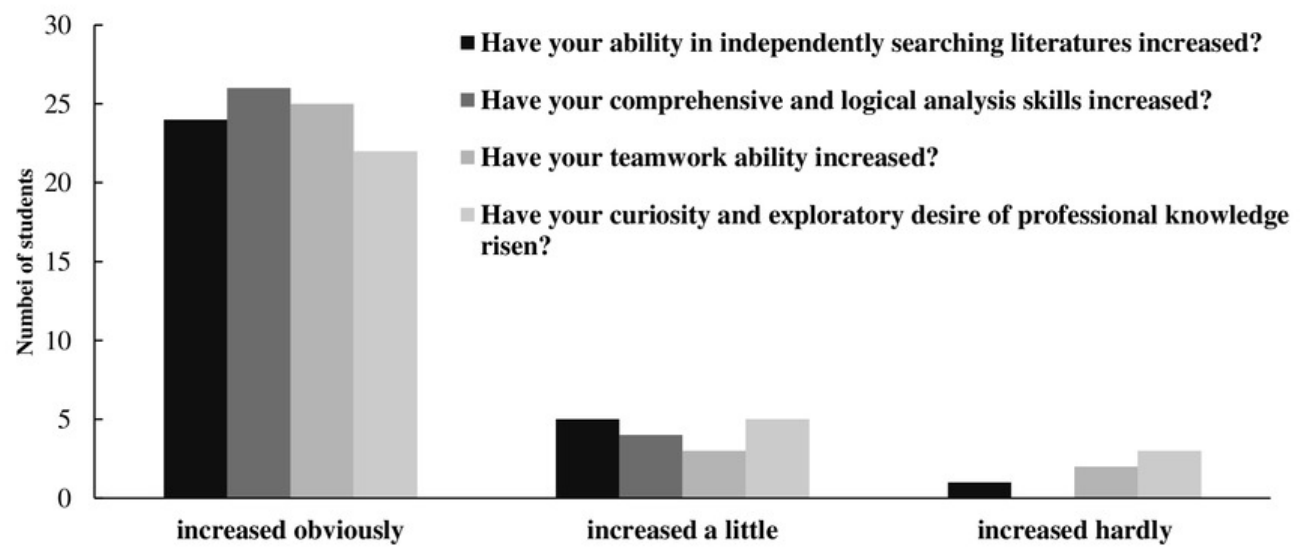


Figure 2

Student responses to the questions about the change in their special abilities in treating dental diseases or communicating with patients after the clinical-simulation PBL training

The figure shows that student responses to the questions about the change in their special abilities in treating dental diseases or communicating with patients after the clinicalsimulation PBL training 


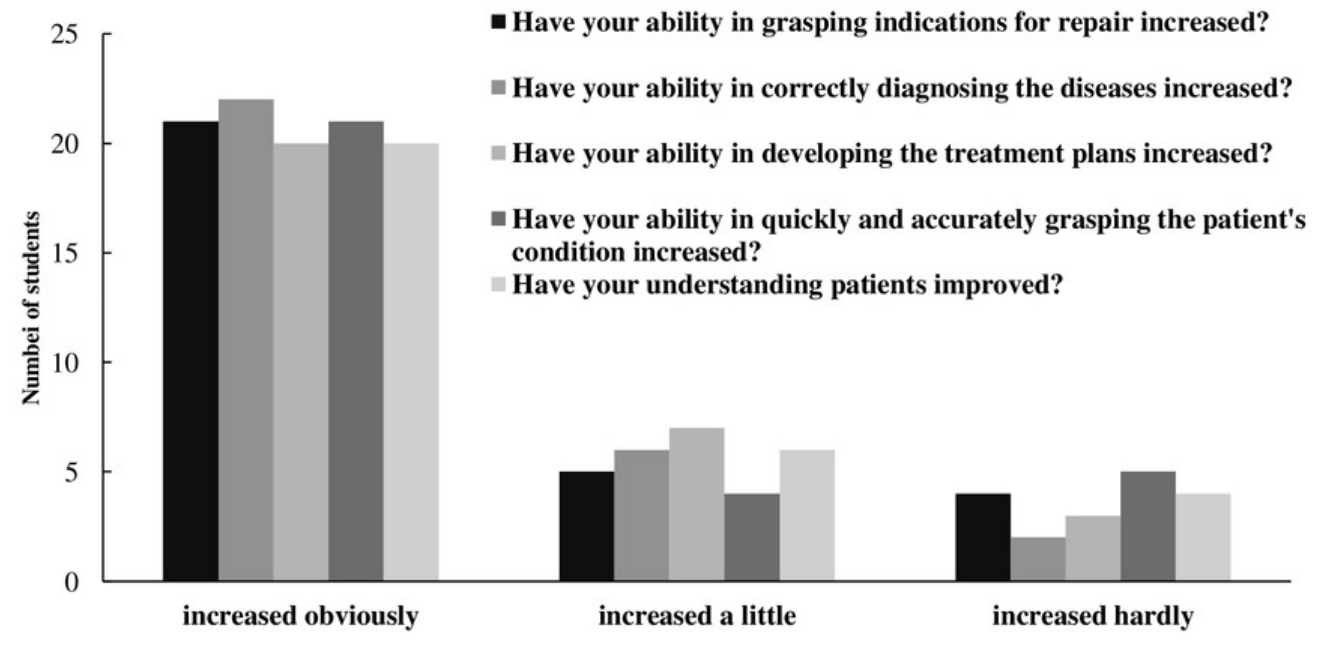


Figure 3

Student responses to the questions about their cognition of the teaching method

The figure 3 shows that student responses to the questions about their cognition of the teaching method 


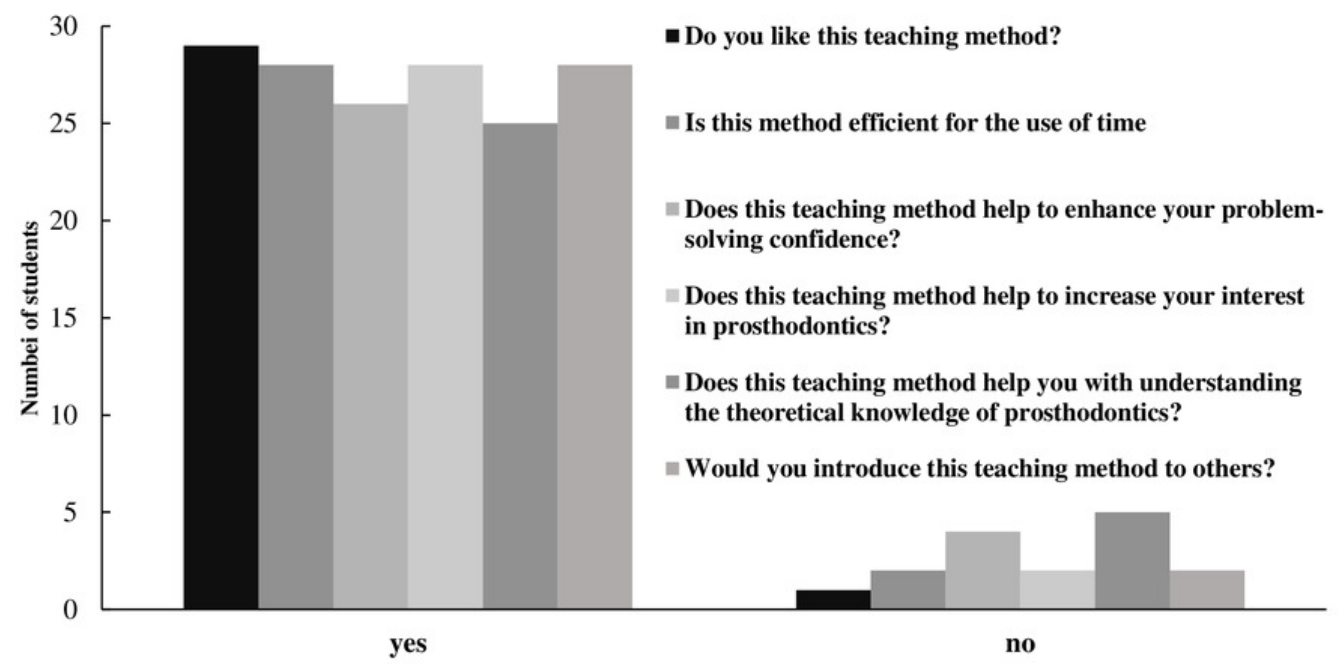


Figure 4

Student responses to the question: How did you find the value of this teaching method, as compared with that of the traditional teaching?

The figure4 shows that student responses to the question: How did you find the value of this teaching method, as compared with that of the traditional teaching? 


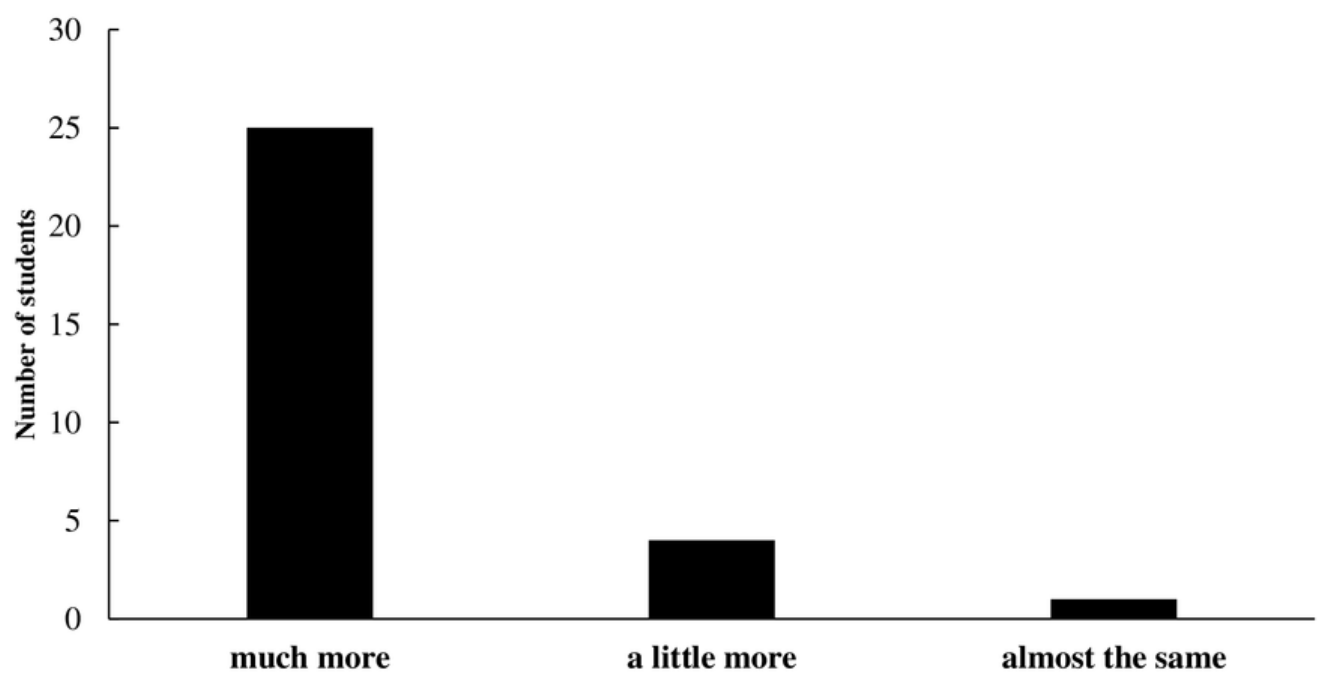


Figure 5

Paper examination results of the clinical-simulation PBL training group and the control group

The figure shows that paper examination results of the clinical-simulation PBL training group and the control group 


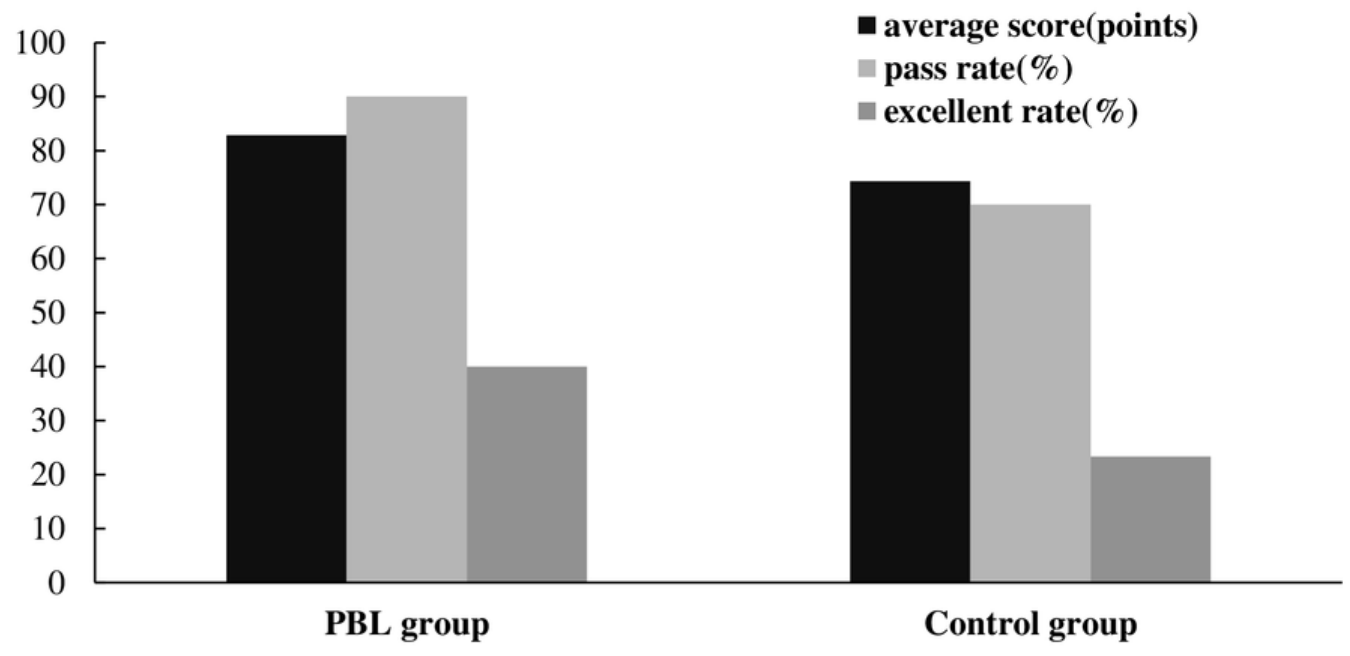




\section{Table 1 (on next page)}

Score sheet for clinical practice

The table shows that score sheet for clinical practice 
1. Whether the appearance of the students meets the hygiene requirements. For example, whether the hat, mask, and glove are worn correctly.

2. Whether the students' attitude is pleasant when they face patients, and whether the speed of their speech is appropriate.

3. Whether the inquiry is detailed, whether the purpose and requirements of patients are understood, and whether the patients' urgent issues to be addressed and comprehensive history, including the history, drug allergies, etc., are collected.

4. When conducting oral preliminary examination, whether compliance with aseptic conditions is satisfactory, whether the mouth pulling action is gentle, and whether the chair position is appropriate.

5. Whether the oral examination is complete, comprehensive, includes a related repair inspection, includes the abutments, the gaps of missing teeth, the alveolar ridge and mucosa, occlusion, etc., and examination of other dental, periodontal, and mucosal conditions

6. Whether the auxiliary check is reasonable and comprehensive, whether the diagnosis of oral diseases is accurate and complete, and whether a reasonable differential diagnosis is conducted.

7. Whether the preliminary diagnosis is correct, whether the explanation of the oral condition is sufficiently detailed, and whether several possible treatment plans are developed, including any necessary collaborative treatments involving other departments.

8. Whether a reasonable treatment plan has been determined and described in detail 
to the patients, including the desired treatment time, costs, possible problems, etc.

9. Whether the case history record is comprehensive and standardized.

10. Whether the patients' recognition and satisfaction are received. 


\section{Table 2 (on next page)}

Score results of the clinical simulation in the PBL training group and control group

The table shows that score results of the clinical simulation in the PBL training group and control group 


\begin{tabular}{lll}
\hline Items & $\begin{array}{l}\text { Scores of the clinical simulation } \\
\text { PBL training group } \\
\text { (points) }\end{array}$ & $\begin{array}{l}\text { Scores of } \\
\text { the control group } \\
\text { (points) }\end{array}$ \\
\hline 1 & $4.63 \pm 0.61^{*}$ & $3.45 \pm 0.53$ \\
2 & $4.33 \pm 0.61^{*}$ & $3.12 \pm 0.61$ \\
3 & $14.17 \pm 0.38^{*}$ & $9.27 \pm 1.46$ \\
4 & $4.47 \pm 0.63^{*}$ & $2.87 \pm 0.63$ \\
5 & $14.53 \pm 0.51^{*}$ & $11.12 \pm 1.27$ \\
6 & $9.00 \pm 0.95^{*}$ & $8.03 \pm 0.67$ \\
7 & $9.03 \pm 0.76^{*}$ & $7.00 \pm 0.31$ \\
8 & $14.73 \pm 0.45^{*}$ & $12.20 \pm 0.85$ \\
9 & $4.47 \pm 0.63^{*}$ & $3.41 \pm 0.50$ \\
10 & $9.53 \pm 0.63^{*}$ & $6.80 \pm 0.41$ \\
Total points & $88.90 \pm 2.29^{*}$ & $67.13 \pm 2.20$ \\
\hline
\end{tabular}

2 P.S.: Two groups of comparison $* \mathrm{p}<0.05$ 